\title{
Ganglion Impar Pulsed Radiofrequency for Intractable Coccydynia
}

\author{
Redouane Mecharnia1, Salim Al Lahham²*, Rehan Zahid², Ruba Sada², Saif Badran², Ahmed Mofeed Mokhalalati², \\ Shyias Mohammedali², Mutaz Abuelgasim² and Talal Al Hermit ${ }^{2}$
}

${ }^{1}$ Anesthesia \& Pain Management, Hamad Medical Corporation, Qatar

${ }^{2}$ Plastic surgeon, Hamad medical corporation, Qatar

*Corresponding author: Salim Al lahham, Plastic surgeon, Hamad medical corporation, Qatar

\begin{abstract}
Patient with coccydynia are often not easy to manage, the aim of this single case study was to describe the outcome of ganglion impar pulsed radiofrequency (PRF) of patient with intractable coccydynia. A 31 years old presented with severe coccydynia, symptom provocation occurred during long sitting, pain reached high level (NRS: 8/10). MRI showed degenerative changes in the sacrococcygeal junction. A first session of impar ganglion PRF for 8 min was applied and the patient was improved $80 \%$ for few months (NRS: 2-3/10), although the results of this case report cannot be generalized, it describes the successful outcome of patient with severe coccydynia.
\end{abstract}

Keywords: Coccydynia; Impar ganglion; Pulsed radiofrequency

\section{Introduction}

Ganglion impar is the lowest paravertebral sympathetic chain, which is placed at the anterior aspect of sacrococcygeal disc used to be blocked in many chronic pain conditions originating from pelvic structures such as the coccyx. Coccydynia is one of chronic pain syndrome which is usually intractable to known therapeutic modalities such as medications, steroid injection and physical therapy manipulation, in addition, there are invasive approaches such as neurolysis with alcohol, phenol or ablative radiofrequency, these approaches are neurodestructive techniques. PRF is a neuromodulative application that has been used for years for chronic pain syndromes. We present a case of coccydynia successfully treated with PRF for neuromodulation of the ganglion impar.

\section{Patient Description}

A 31-year-old man healthy, working as software developer, had pain over the coccyx started in 2015 without any history of trauma, increasing with time. The pain triggered with the prolonged sitting which made his work very difficult and start thinking to change his job, seen by many doctors, MRI of spine coccyx requested concluded degenerative changes in the sacrococcygeal junction Figure 1, he had been diagnosed as coccydynia and treated with conservative management including medications, coccyx steroid infiltrations and physical therapy manipulations, after the failure of conservative methods patient was referred to our pain clinic by his orthopedist, at this stage he became depressed because of functional limitation and job problems he described the pain as $8 / 10$ point on verbal numerical rating scale (NRS). The clinical examination found the pain originated from coccyx. The ganglion impar block test was explained to the patient, done with lidocaine $1 \%(5 \mathrm{ml})$ which came positive, so patient planned for PRF of ganglion impar. He was admitted to the operating theater in prone position on the table, with a pillow under the anterior superior iliac spine to flatten the normal lumbar lordosis. First, intravenous access was inserted, and monitors were attached. The midline of the sacrococcygeal area was cleaned with antiseptic and sterile drapes were placed. Lidocaine $1 \%(5 \mathrm{~mL})$ was used for skin infiltration over the coccyx. A 22-gauge RF needle $10 \mathrm{~cm}$ (Cosman electrode CU TM) with an active tip of $5 \mathrm{~mm}$ was inserted through the sacrococcygeal disc to the anterior 
surface of the coccygeal disc, with the guidance of anteroposterior and lateral fluoroscopy view of the sacrum and coccyx. After negative aspiration, the fluoroscopic image was observed as $2 \mathrm{~mL}$ of nonionic radiocontrast was injected to confirm the good placement of the needle Figure 2. Before applying PRF, sensory and motor tests were checked, which showed a sensory response at $0.35 \mathrm{~V}(50 \mathrm{~Hz})$, a motor response below $2 \mathrm{~Hz}$. The treatment was maintained for $4 \mathrm{~min}$ twice when the temperature at the needle tip did not exceed 42C. The total treatment time was $8 \mathrm{~min}$. He described the pain as zero on the NRS post procedure. After the procedure, the patient kept under close monitoring to check for any complications. Five months later, patient improved almost $80 \%$ and he was asked to describe the pain once more, and it was within two to three points NRS [1-4].

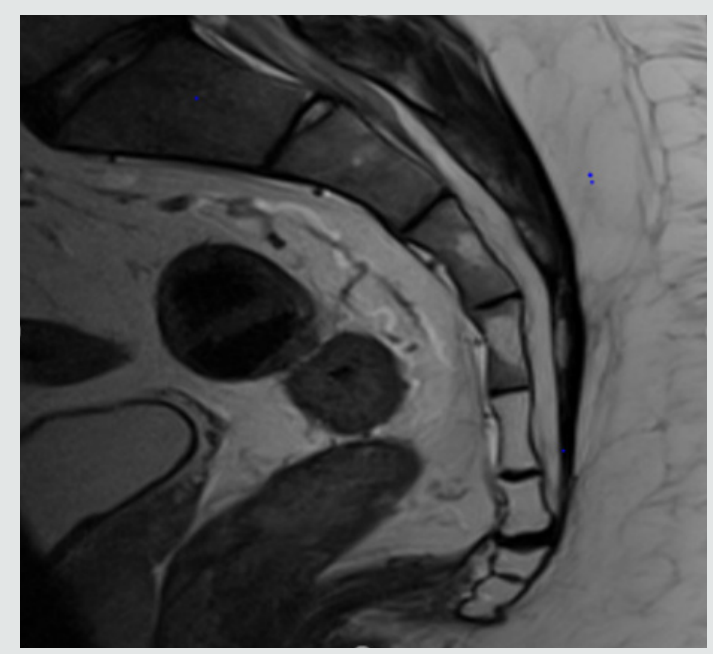

Figure 1: MRI of spine coccyx: degenerative changes of sacrococcygeal junction.

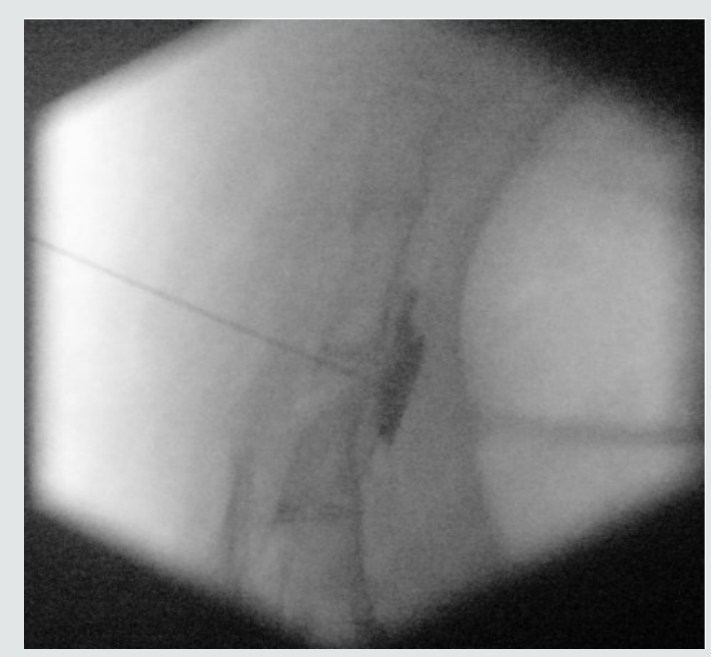

Figure 2: Impar ganglion PRF: trans-sacrococcygeal needle placement with contrast injection.

\section{Comment and Discussion}

Coccydynia is one of chronic pain syndrome difficult to manage, adversely affects quality of life, and the outcome of conservative methods is very poor. There are trials showed that PRF application is not only effective but also safe for nerve tissue because of its application of 42C. There are also other choices in the relief of coccydynia, such as ablative RF application and coccygectomy. These methods are more invasive and have higher risks of adverse effects compared with PRF application. Pulsed radiofrequency as new alternative practice in chronic pain is not widely used in the management of coccydynia and We believe that the transsacrococcygeal disc approach using PRF for ganglion impar block is not only simple but also effective for the treatment of coccydynia [5-8].

\section{References}

1. Mitra R, Cheung L, Perry P (2007) Efficacy of fluoroscopically guided steroid injections in the management of coccydynia. Pain Physician10: 775-781.

2. Cahana A, Van Zundert J, Macrea L, Van Kleef M, Sluijter M (2006) Pulsed radiofrequency: current clinical and biological literature available. Pain Med 7(5): 411-423.

3. Podhajsky RJ, Sekiguchi Y, Kikuchi S, Myers RR (2005) The histologic effects of pulsed and continuous radiofrequency lesions at $42 \mathrm{C}$ to rat dorsal root ganglion and sciatic nerve. Spine 30(9): 1008-1013.

4. Sluijter M, Racz G (2002) Technical aspects of radiofrequency. Pain Pract 2: $195-200$.

5. Hodges SD, Eck JC, Humphreys SC (2004) A treatment and outcomes analysis of patients with coccydynia. Spine 4(2): 138e140.

6. Toshniwal GR, Dureja GP, Prashanth SM (2007) Transsacrococcygeal approach to ganglion impar block for management of chronic perineal pain: a prospective observational study. Pain Physician 10(5): 661-666.

7. Usta B, Gozdemir M, Sert H, Muslu B, Demircioglu RI (2010) Fluoroscopically guided ganglion impar block by pulsed radiofrequency for relieving coccydynia. J Pain Symptom Manage 39(6): e1-2.

8. Postacchini F, Massobrio M (1983) Idiopathic coccygodynia. Analysis of fifty-one operative cases and a radiographic study of the normal coccyx. J Bone Joint Surg Am 65(8): 1116-1124. 
This work is licensed under Creative Commons Attribution 4.0 License

To Submit Your Article Click Here: Submit Article

DOI: $10.32474 /$ GJAPM.2019.01.000102

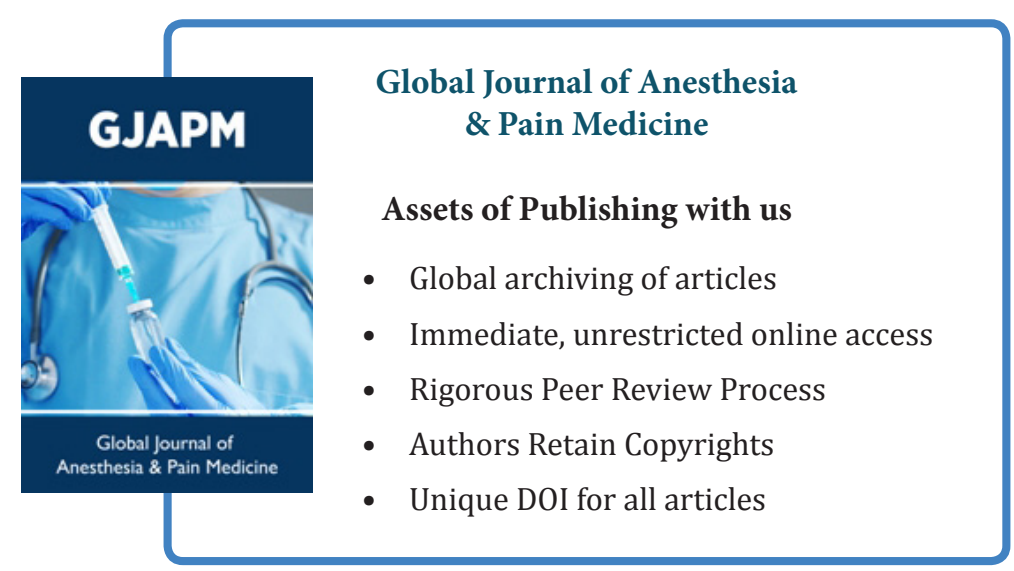

